\title{
HAND CONTROLLER COMMONALITY EVALUATION PROCESS
}

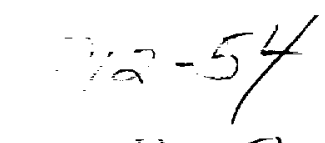

\author{
Mark A. Stuart, John M. Bierschwale, Robert P. Wilmington, \\ Susan C. Adam, and Manuel F. Diaz \\ Lockheed Engineering and Sciences Company
}

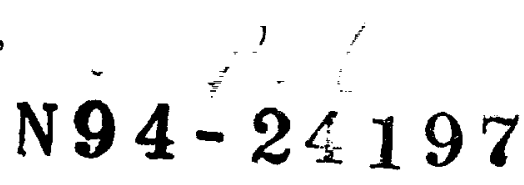

Dean G. Jensen

NASA Lyndon B. Johnson Space Center

\section{INTRODUCTION}

Hand controller selection for NASA's Orbiter and Space Station Freedom is an important area of human-telerobot interface design and evaluation. These input devices will control remotely operated systems that include large crane-like manipulators (e.g., Remote Manipulator System or RMS), smaller, more dexterous manipulators (e.g., Flight Telerobotic Servicer or FTS), and free flyers (e.g., Orbital Maneuvering Vehicle or OMV). Candidate hand controller configurations for these systems vary in many ways: shape, size, number of degrees-of-freedom (DOF), operating modes, provision of force reflection, range of movement, and "naturalness" of use. Unresolved design implementation issues remain, including such topics as how the current Orbiter RMS rotational and translational rate hand controllers compare with the proposed Space Station Freedom hand controllers, the advantages that position hand controllers offer for these applications, and whether separate hand controller configurations are required for each application.

Common Space Station and Orbiter hand controllers are desirable for many practical reasons. Common hand controllers would reduce the negative transfer that could occur if many different types of hand controllers were used. The hand controllers need to be selected to minimize astronaut training requirements. Other considerations include the number of controllers required if each system had unique controllers and the associated weight and volume required to accommodate multiple sets and spares.
Several previous studies have evaluated operator performance differences caused by using different hand controller configurations during remote manipulation tasks. For example, O'Hara (1987) compared bilateral force-reflecting replica master controllers to proportional rate six degrees-of-freedom (DOF) controllers during dual-armed remote manipulation tasks and discovered several differences. The six-DOF rate controllers were rated significantly higher in cognitive workload and manual-control workload (ability to control the end effector and the equipment) during dual-armed tasks. O'Hara also reported that the force-reflecting master controller was rated significantly higher in physical workload compared to the six-DOF rate controller. In conclusion, O'Hara found that master controllers resulted in lower performance times and allowed more "natural" control, while sixDOF rate controllers were lower in physical workload. This study was significant, yet limited because only two hand controller types were evaluated under limited operating conditions.

Another relevant study conducted by Honeywell (1989) described current hand controller concepts, the hand controller configurations proposed for Space Station Freedom, and the requirements of the space station systems that will use hand controllers. Much of the report was based upon a survey administered to industrial participants, NASA, and universities. A third study (Stuart, Smith, Bierschwale, and Jones, 1989) evaluated the anthropometric and biomechanical interface between test subjects and three and six-DOF joystick and mini-master hand controllers and found that subjects can experience various 
types of muscle discomfort due to certain hand controller features. Since these two reports contain little empirical hand controller task performance data, a controlled study is needed that tests Space Station Freedom candidate hand controllers during representative tasks. This study also needs to include anthropometric and biomechanical considerations.

\section{EVALUATION}

The NASA hand controller commonality evaluation objective was to recommend the hand controller configuration(s) that can meet the Space Station requirements while accomplishing optimal control of each particular system. The recommended configuration(s) shall be chosen to maximize performance, minimize training, and minimize cost of providing safe and productive controls for the Space Station Freedom crew.

The hand controller commonality evaluation was conducted as three separate experiments. Experiment One was a non-astronaut hand controller evaluation at three test facilities. Experiment Two was an astronaut hand controller evaluation at the same three test facilities. Experiment Three was a hand controller volumetric evaluation done primarily in the Orbiter and Space Station mockups. All of the evaluations took place at NASA Johnson Space Center (JSC).

\section{EXPERIMENT ONE METHODS}

Experiment One was conducted as a repeated measures evaluation (within-subjects design) for each of the six tasks evaluated. These tasks are described in the Apparatus section below. Test subjects used all of the hand controllers for their respective tasks in those modes supported by the hand controllers and the facilities.

\section{SUBJECTS}

Twenty-four non-astronaut test subjects were used in Experiment One. Test subjects were partitioned into six independent groups of four test subjects. Each test subject group performed one of the six remote manipulation tasks. Twelve test subjects who had prior dexterous manipulator experience formed three groups, eight test subjects who had prior RMS simulation experience formed two groups, and four test subjects who had prior free flyer experience formed one group.

\section{APPARATUS}

Physical Simulations. Physical simulations were performed in the Remote Operator Interaction Laboratory (ROIL). These consisted of the following tasks: fluid quick-disconnect coupling; simulated ORU change-out; and thermal insulation blanket removal. These tasks were performed using a Kraft manipulator slave arm with a JR3 force-torque sensor.

Computer Simulations. Computer simulations took place at two different test sites - the Systems Engineering Simulator (SES) and the Displays and Controls Laboratory (D\&CL). The SES tasks were used to investigate rate control mode hand controller characteristics while controlling dynamic free flyer and Space Station Remote Manipulator System (SSRMS) simulations. The specific tasks were OMV docking and logistics module transfer.

The D\&CL tasks were used to investigate hand controller characteristics during rate mode for a crane-type manipulator and both rate and position modes for a dexterous manipulator (both kinematic simulations). The D\&CL task consists of a sequential SSRMS/dexterous manipulator operation (SSRMS used as a transport device) to perform an ORU replacement task.

Hand Controllers Evaluated. Hand controllers evaluated in this study were provided by NASDA of Japan, McDonnell Douglas/ Honeywell, the Canadian Space Agency, and Goddard Space Flight Center. These hand controllers are illustrated and described in Figure 1. 


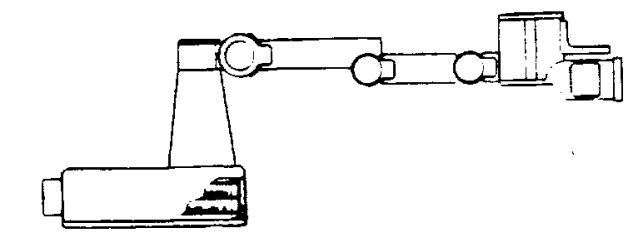

SCHILLING OMEGA 6-DOF

(Rate, position, non-force reflecting and force reflecting mini-master)
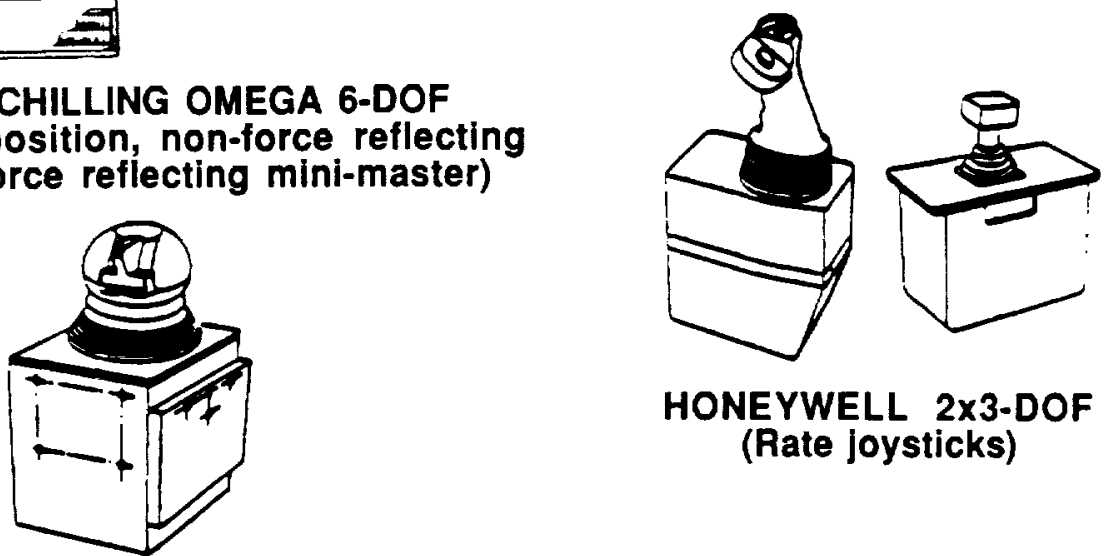

HONEYWELL 2×3-DOF (Rate joysticks)

CAE 6-DOF (Rate joystick)
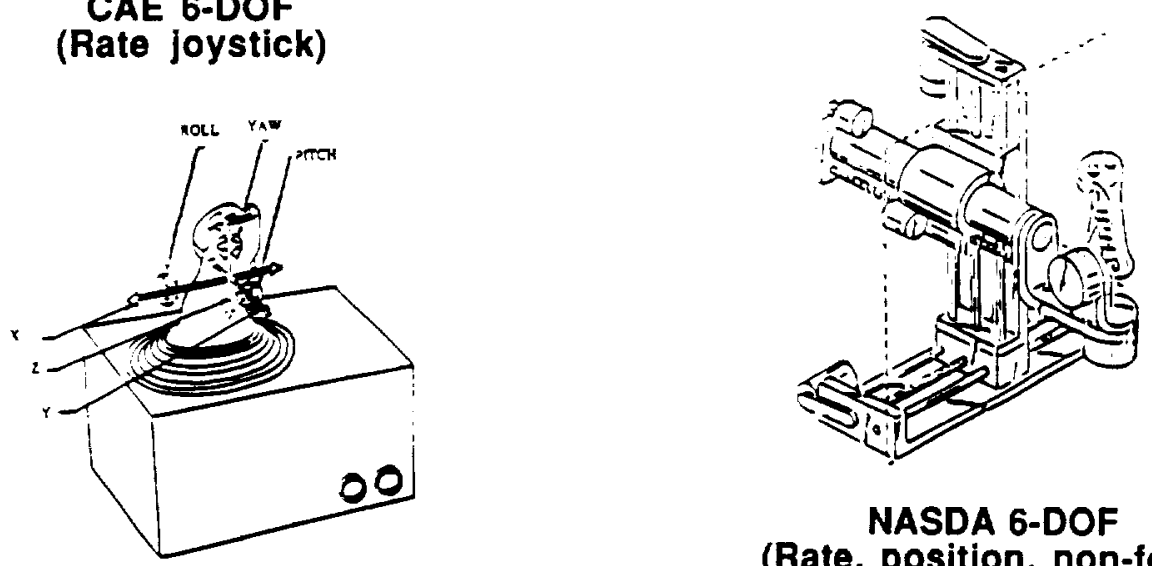

NASDA 6-DOF

HONEYWELL 6-DOF

(Rate, position, non-force reflecting and force reflecting)

(Rate, position, non-force reflecting and force reflecting joystick)
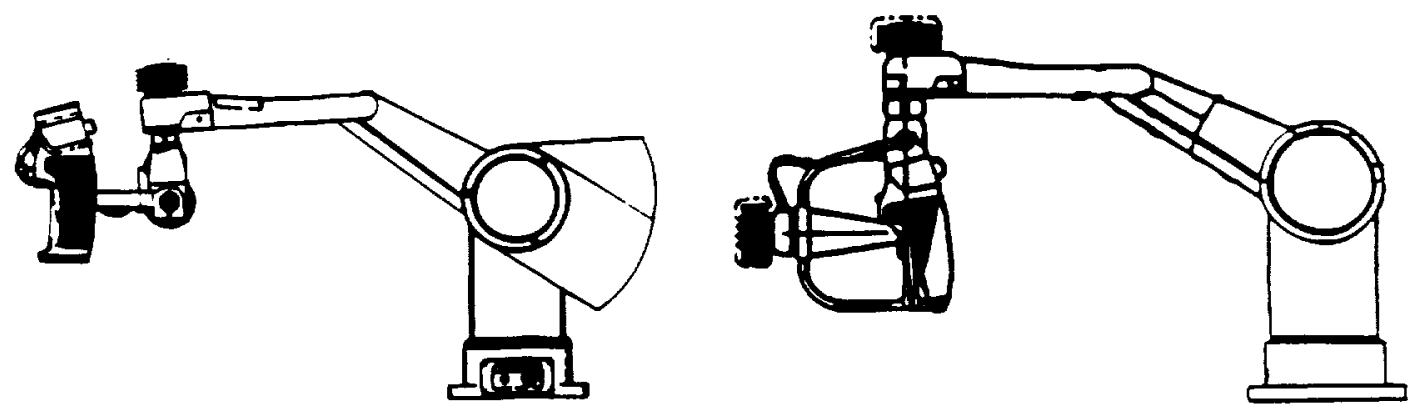

KRAFT NATIVE 6-DOF (Position, force reflecting mini-master)

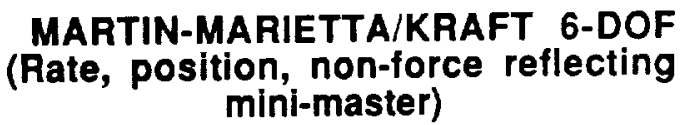

Figure 1. Illustrations and characteristics of hand controllers evaluated. 


\section{PROCEDURE}

The procedure at each test site included pilot testing with operations-experienced test subjects. At each of the three test sites, test subjects were allowed to switch between different camera views as well as use fineadjustment camera controls such as focus, pan, tilt, and zoom. The switching and adjusting was done by the test administrator. Tasks at all test sites were broken into their respective subtasks for performance analysis purposes.

ROIL. Test subjects within each of the three dexterous manipulator-experienced groups performed one of the three physically simulated tasks. The ROIL tested position mode with no force reflection (hapticproprioceptic), position mode with force reflection, and rate mode while operators used a dexterous manipulator. The test subjects followed a prescribed procedure during the performance of the three physical simulation tasks. The subjects used predetermined camera positions of the remote worksite. One of the cameras provided a global view of the entire taskboard area. Camera positions were optimized per task prior to data collection. Test subjects received an equal amount of laboratory training with each of the hand controllers before data collection began. After receiving training for a specific hand controller, each test subject performed the task two times with that controller. The procedure continued in this fashion until subjects within each group performed their respective task twice while using each of the hand controllers. Hand controller use was counterbalanced to control for order effects. After completing two task trials using each hand controller, each subject was administered questionnaires to collect subjective data.

$S E S$. Test subjects within one of the RMSsimulation-experienced groups performed the logistics module transfer task and test subjects within the free-flyer-experienced group performed the OMV task. The SES tested the controllers in rate mode while test subjects used the SSRMS or the OMV. The OMV was controlled in pulse mode and the SSRMS tasks were controlled using the standard proportional rate mode. The subjects used predetermined simulated camera views of the remote worksite as well as a simulated direct view. Subjects completed a familiarization session prior to data collection in the SES and also performed the simulated task two times with each hand controller. Questionnaires were administered after performance of the tasks.

$D \& C L$. Test subjects within the second RMSsimulation-experienced group performed the dual SSRMS/FTS ORU task. The D\&CL tested rate and position (non-force-reflective) while operators used a dexterous manipulator in conjunction with the SSRMS. The SSRMS was controlled in the rate mode and the dexterous manipulator was controlled in both rate and in position mode. The subjects used simulated camera views of the remote worksite. After performing the simulated task two times questionnaires were administered.

\section{DATA COLLECTION}

Task performance data included the following: time to complete each subtask, reach limits, active hand controller time, the number of hand controller inputs, and error or accuracy counts. Questionnaires were administered to collect the following types of subjective impressions: general acceptability, mental and physical fatigue, and hand controller suitability for specific tasks.

\section{EXPERIMENT TWO METHODS}

Experiment Two used astronaut test subjects who performed each of the six tasks at all three test sites.

\section{SUBJECTS}

Six crewmembers were used as test subjects in this phase of the evaluation. Prior hand controller experience of each crewmember was assessed.

\section{PROCEDURE}

Familiarization with the tasks was required before the crew evaluation took place. This 
varied according to the experience level of each individual crewmember. For example, somewhat more familiarization time was necessary for those crewmembers who had no prior OMV or dexterous manipulator task experience.

Each crewmember performed a structured subset of each of the six tasks described in the Experiment One Methods Section. During the task, performance data, such as speed and accuracy, were collected from each crewmember. After performing the structured subset of each of the six tasks with each hand controller, the crewmember was given a brief questionnaire to fill out.

\section{EXPERIMENT THREE METHODS}

Experiment Three was a volumetric evaluation which involved astronaut test subjects using all of the hand controllers.

\section{TEST SUBJECTS}

Four astronauts performed the evaluations. Attempts were made to have test subjects that range in body sizes from the 95th percentile male to the 5 th percentile female (workstations are required to accommodate this range of users).

\section{APPARATUS}

Hand controller volumetric evaluations were performed in the Space Station, Cupola, and Shuttle mockups located at NASA JSC. Hand controllers evaluated in Experiment Three are listed in the Experiment One Apparatus section.

\section{PROCEDURE}

Single and dual hand controller usage for one operator was addressed at the command and control workstation and the cupola workstation. Side-by-side operator operation was addressed in the cupola. Hand controller mounting and adjustment in the Space Station and Cupola mockups were achieved using two tripods.

\section{DATA MEASUREMENT}

Data were collected with both a video recorder and a $35 \mathrm{~mm}$ camera. Hand controller locations for the various subjects were also recorded. The evaluations consisted of questionnaire administration and anthropometric data collection that addressed the following issues: hand controller swept volume; operator/workstation placement (e.g., crew movement ability in the area); display viewing characteristics (e.g., line of sight characteristics, display obstruction from hand controllers); and reach envelope characteristics (e.g., ability to reach workstation controls). The anthropometric data were incorporated into an analysis of each hand controller configuration within the appropriate workstations on Space Station.

\section{RESULTS}

Results of data analyses are summarized as follows: no appreciable astronaut/nonastronaut differences on the performance and subjective data collected; subjective data supported objective (performance) data; trends were consistent across all three tasks conducted; rate control-mode was consistently superior to position control-mode; no advantage demonstrated for force reflection; joystick controllers were superior to minimaster controllers; and the $2 \times 3$ DOFs, CAE, and the Honeywell rate-mode were consistently the top hand controller configurations. As a result of these evaluations, a $2 \times 3$-DOF hand controller configuration was decreed the Space Station Freedom baseline configuration.

\section{REFERENCES}

1. Honeywell, Inc. (1989). Hand controller commonality study -- 289. 16007. Clearwater, Florida.

2. O'Hara, J. (1987). Telerobotic control of a dextrous manipulator using master and six-DOF hand controllers for space assembly and servicing tasks. Proceedings of the Human Factors 
Society 31st Annual Meeting. Santa

Monica, CA: Human Factors Society.

3. Stuart, M. A., Jones, S. F., Smith, R. L., and Bierschwale, J. M.

Preliminary hand controller evaluation. Houston, TX: NASA Lyndon B. Johnson Space Center, 1989; NASA Tech. Report JSC-23892. 